\title{
Digital 3D Modeling of vestment Based on Project-camera System
}

\author{
Jun Tao \\ School of Mathematics and Computer Science \\ Jianghan University, \\ Wuhan, Hubei, P.R.China 430056
}

\begin{abstract}
With the coming of information age and the development of computer science, digitalization of whole vestment is becoming more and more important. The surface of whole vestment is sequent and glossy so that it is lack of the texture characteristic which is the key of digital 3D modeling. According to this reason, the structure illumination is steered into a method of this paper. The paper proposes the method by which 3D model of whole vestment is created from 2D image sequences directly but not by the common techniques using general CAD model. In the paper the structure illumination is generated by the slide projector and the modeling of whole vestment is based on the strict theory of the digital photogrammetry, computer vision and image processing pattern recognition. Because whole vestment is lack of the applicable texture for matching as above said, the characteristic texture generated by the structure illumination is added onto the surface of whole vestment. After the characteristic texture is extracted from images and is matched well, 3D coordinates of the characteristic texture can be calculated out by the space forward intersection. Then the whole vestment model is acquired by connecting all neighbour space points in the TIN and rendering the real texture of whole vestment automatically. The 3D modeling method is untouched so that it is nondestructive which is just suitable for the messaline and the clothing. The method of whole vestment 3D modeling proposed in the paper is flexible, effective and practical, which is confirmed by the results of the reconstructing experiments.
\end{abstract}

Keywords-Vestment Digitalization; 3D Modeling; Structure Illumination; Slide Projector; Digital Photogrammetry

\section{INTRODUCTION}

As the development of production and design of whole vestment in the information age, the digitalization of whole vestment becomes more and more important and necessary in the field of textile. The digitalization of whole vestment provides the convenience and the advantage for the design and the production of whole vestment.

The digitalization of whole vestment emphasizes on the 3D model of it. Digital 3D modeling is referred as a process of the recovery of three-dimensional model from the twodimensional digital camera images [1]. During the image formation process of the camera, explicit 3D information about the scene or objects in the scene is lost. Therefore, 3D model or depth information has to be inferred implicitly from the 2D intensity images. The key of the traditional method lies in the matching of the corresponding features in the images. When there is no feature or lack of feature in the objects, or when the features cannot be matched correctly at all, the main problems appear in the process of the $3 \mathrm{D}$ modeling [2].

The surface of whole vestment is sequent and glossy so that it is lack of the texture characteristic which is the key of digital 3D modeling. According to this reason, the structure illumination is steered into a method of this paper. The paper proposes the method by which 3D model of whole vestment is created from 2D image sequences directly but not by the common techniques using the general CAD model. In the paper the structure illumination is generated by the slide projector and the modeling of whole vestment is based on the strict theory of the digital photogrammetry, computer vision and image processing pattern recognition. The 3D modeling method is untouched so that it is nondestructive which is just suitable for the messaline and the clothing.

\section{EQUIPMENTS AND METHODOLOGY}

\section{A. Whole equipments}

The whole equipments are composed of a slide projector, a digital camera, a control ground and a computer. The computer controls the other three equipments working together automatically and efficiently. The control ground is a planar grid on a rotating platform. The planar grid is functioned as the calibration of the slide projector and the digital camera. The digital camera mainly takes images of whole vestment and the slide projector supplies the structure illumination for the surface of whole vestment. After calibrated respectively in advance, the whole equipments are similar with the binocular vision system on the principle of $3 \mathrm{D}$ reconstruction.

The whole equipments are easy to gain relatively and can be connected together and settled down conveniently and flexibly. The figure of whole equipments is shown on the Figure 1 as following.

\section{B. Algorithm of the method}

The collinear equations are formula 1 as the main and common formula in the algorithm of the method $[7,8]$.

$$
\begin{aligned}
& x=-f \frac{a_{1}\left(X-X_{s}\right)+b_{1}\left(Y-Y_{s}\right)+C_{1}\left(Z-Z_{s}\right)}{a_{3}\left(X-X_{s}\right)+b_{3}\left(Y-Y_{s}\right)+C_{3}\left(Z-Z_{s}\right)}, \\
& y=-f \frac{a_{2}\left(X-X_{s}\right)+b_{2}\left(Y-Y_{s}\right)+C_{2}\left(Z-Z_{s}\right)}{a_{3}\left(X-X_{s}\right)+b_{3}\left(Y-Y_{s}\right)+C_{3}\left(Z-Z_{s}\right)},
\end{aligned}
$$


where $x_{0}, y_{0}, f=$ the intrinsic parameters of the projector

$X_{S}, Y_{S}, Z_{S}=$ the coordinates of the projector

centre

$X, Y, Z=$ the space coordinates of points

$x, y=$ the image coordinates of the relative

points

$$
R=\left\{a_{i}, b_{i}, c_{i}, i=1,2,3\right\}=\text { the rotated matrix }
$$

made up of rotated angles

To the planar grid, the coordinates $Z=0$

There are four equations listed ordinarily according to a pair of homologous points. However, the space coordinates $(\mathrm{X}, \mathrm{Y}, \mathrm{Z})$ can be calculated out only by three equations. Through the line matching based on the structure illumination, there exist three known equations shown in the Figure 2.

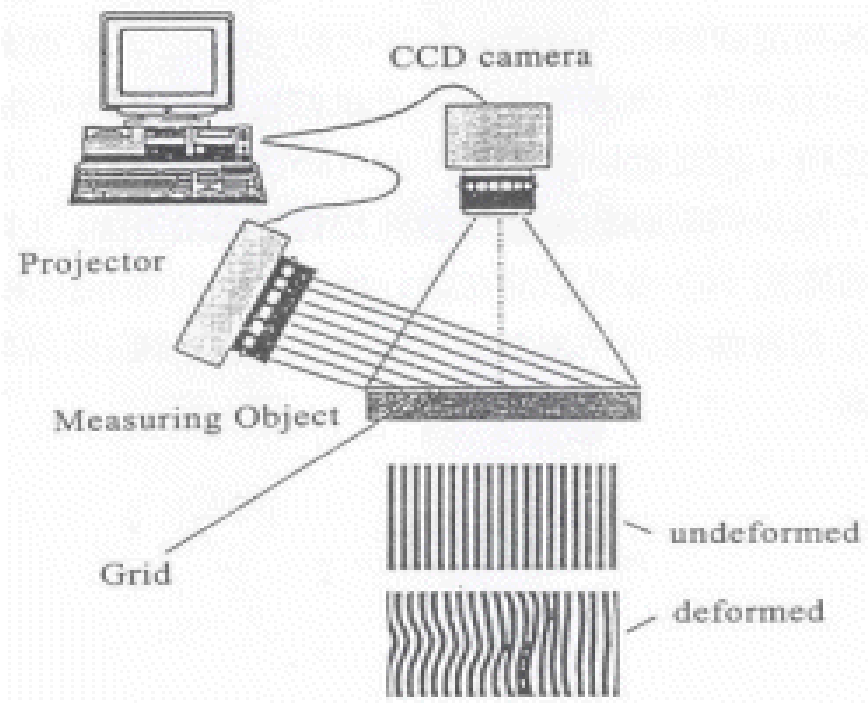

Figure 1. The principle of whole equipments

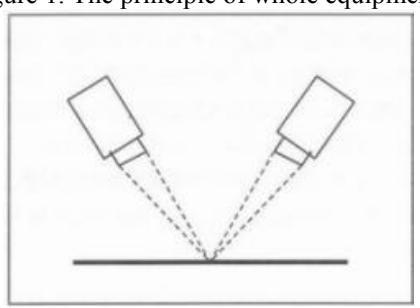

Figure 2. The line match based on the structure illumination

\section{Calibration of the digital camera}

The digital camera needs to be calibrated in advance, because the late computation of the coordinates of the space feature requires the intrinsic and extrinsic parameters of the digital camera. Therefore, the calibration of the digital camera is an important preceding step.
Direct Linear Transformation (DLT) is a well-known method used in close-range photogrammetry because of it's no need for initial value of camera intrinsic and extrinsic parameters. The existed camera calibration techniques are studied thoroughly. The restricting condition among 2DDLT parameters is worked out using the correspondence of collinear equation and 2D-DLT. The decomposition of initial values of camera intrinsic and extrinsic parameters using 2D-DLT is detailed. Planar-scene camera calibration algorithm with collinear equations is addressed. So the calibration of the digital camera is accomplished entirely and the intrinsic and extrinsic parameters of the digital camera are ready to be used in the future $[3,4]$.

If the camera is not an ordinary one but a special digital measuring-camera, the calibration could be cancelled because the intrinsic parameters of the digital camera can be gotten as the known data. However, the extrinsic parameters of the digital camera are still required to calculate out first.

\section{Calibration of the ordinary projector}

In this approach proposed in the paper, the slide projector makes the same function as a digital camera. So the ordinary slide projector also desires to be calibrated in advance. Its intrinsic and extrinsic parameters are also applied to the computation process.

The algorithm with 2D direct linear transformation (2D-DLT) and collinear equations is used to calibrate the projector. The algorithm is addressed systematically and entirely as following. First, the image coordinates of the projector are designed carefully and the space coordinates of the projector are computed by the image data and the intrinsic and extrinsic parameters of the digital camera. Then, the decomposition of initial values of the projector intrinsic and extrinsic parameters using the correspondence of 2D-DLT and collinear equation is deduced. Finally, the projector calibration parameters are worked out by the whole adjustment. By this time, the intrinsic and extrinsic parameters of the ordinary slide projector are ready to be used in the next program $[5,6]$.

\section{DATA AND EXPERIMENTAL RESULTS}

The size of the rotating platform is $60 \mathrm{~cm} \times 60 \mathrm{~cm}$. A planar grid is fixed upon it, which supplies the controlling points and the coordinates system. There are $18 \times 18=324$ controlling points in the planar grid. The interval of these points is the same and is $30 \mathrm{~mm}$. Each point has its own serial number which is exclusive. By using the coordinates of these points, the digital camera and the slide projector can be calibrated correctly and the extrinsic parameters of both are also calculated out entirely.

The size of the feature slide designed is 1024 pixels $\times 768$ pixels. The texture feature is designed as stripe which is better to show the surface of the industrial parts. The stripe is between white and black and is 7 pixels or 5 pixels or 3 pixels in width respectively. 
When the positions of the camera and the projector are adjusted well and fixed relatively, both need to be focused respectively. The distance from the digital camera to the rotating platform is about 0.8 meters and the distance from the slide projector to the rotating platform is about 1.5 meters.

The whole vestment is put on a model in the centre of the rotating platform. The slide projector projects the stripe in the slide onto the surface of the whole vestment. Then the camera is used to take sequential images of the whole vestment with the slide projector illuminating when the stripe width changes continually. In the experiment the camera takes images from 3 stripe widths and there are 3 images in total as the image data. The size of each image is 1300pixels $\times 1030$ pixels.

According to the method above, all points projected on the surface of whole vestment can be computed out entirely and connected by neighbor relationship. The final digital 3D model of whole vestment is shown on the Figure 3.
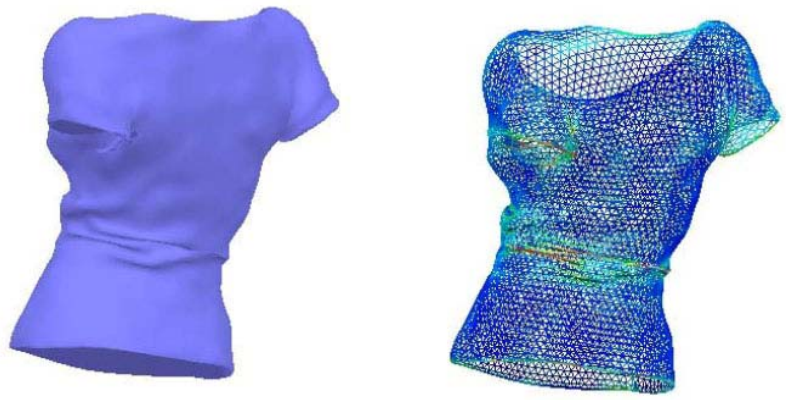

Figure 3. The digital 3D model of vestment

\section{CONCLUSIONS}

The paper proposes a method by which 3D model of whole vestment is created from $2 \mathrm{D}$ image sequences directly but not by the common techniques using the general CAD model. The slide projector supplies the texture characteristic for the surface of whole vestment and the digital camera takes images for the data source. The 3D modeling method is untouched so that it is nondestructive which is just suitable for the messaline and the clothing. The final 3D digital model of whole vestment is good enough for the production and the design of whole vestment. The method of whole vestment $3 \mathrm{D}$ modeling provided in the paper is flexible, effective and practical, which is confirmed correctly by the results of the reconstructing experiments.

\section{ACKNOWLEDGMENT}

Supported by S\&T research fund for planning project of wuhan science and technology bureau (200851799524-08)

Supported by S\&T research fund of wuhan education bureau (2008K044) and

S\&T research fund of Hubei provincial education department (B20104504)

\section{REFERENCES}

[1] Wenhao Feng, Close-Range Photogrammetry[M], Wuhan University Press, Wuhan, China,2002, pp. 268-271.

[2] Deren Li, Analytical Photogrammetry[M], Wuhan University Press, Wuhan, China, 1992, pp. 26-33, pp. 59-61.

[3] Zuxun Zhang, and Yongjun Zhang, Digital Camera Calibration Using 2D-DLT And Collinear Equation With Planar Scenes (in Chinese)[J], Geomatics and Information of Wuhan University, Vol. 27, No. 6, Wuhan, China, 2002, pp. 75-82.

[4] Zhang Zhengyou, A Flexible New Technique for Camera Calibration[A], Technical Report[C], Microsoft Research, Redmond, WA 98052, USA, 1998.

[5] Jianqing Zhang, Jun Tao, Zuxun Zhang, A flexible technique for the slide projector calibration[A], Proceedings of SPIE - The International Society for Optical Engineering[C], Beijing, China, v 5286, n 1, 2003, pp. 187-190.

[6] Jun Tao, Jianqing Zhang, and Zuxun Zhang, Calibration of a Projector with a Planar Grid[A], ISPRS[C], Istanbul, Turkey, 2004.

[7] Zhizhuo Wang, Principles of Photogrammetry[M], Surveying and Mapping Press, Beijing, China,1990, pp. 148-152.

[8] Zuxun Zhang, and Jianqing Zhang, Digital Photogrammetry[M], Wuhan University Press, Wuhan, China, 2000, pp. 112-115.

\begin{tabular}{ccc}
\multicolumn{3}{c}{ TABLE I . THE PARAMETERS OF THE DIGITAL CAMERA AND THE SLIDE PROJECTOR } \\
\hline & Digital Camera & Slide Projector \\
\hline $\mathrm{f} /$ pixel & 3727.227916 & 1855.251838 \\
$\mathrm{x}_{0} /$ pixel & 648.148720 & 555.621441 \\
$\mathrm{y}_{0} /$ pixel & 506.352984 & 473.971580 \\
$\mathrm{Xs} / \mathrm{mm}$ & 91.336315 & -345.013227 \\
$\mathrm{Ys} / \mathrm{mm}$ & -867.369190 & -872.986760 \\
$\mathrm{Zs} / \mathrm{mm}$ & 617.363871 & 762.109676 \\
$\Psi /$ radian & -0.106019 & 0.459701 \\
$\Omega /$ radian & 0.925349 & 0.832287 \\
$\mathrm{~K} /$ radian & 0.132368 & -0.391013
\end{tabular}

